\title{
Fusion of Infrared and Visible Light Images Based on Compressive Sensing
}

\author{
Yanhai $\mathrm{Wu}^{1}$, Ye Zhang ${ }^{1}, \mathrm{Nan}_{\mathrm{Wu}}{ }^{2}$, Jing Wang ${ }^{1}$ \\ ${ }^{1}$ School of Telecommunication and Information Engineering, Xi'an University of Science and \\ Technology, Xi'an,710054,China \\ ${ }^{2}$ Department of Computer Science and Technology, Shaanxi Xueqian Normal University, \\ Xi'an,710061, China
}

Keywords: Image fusion; Compressive Sensing; NSCT; Infrared Images; Visible Light Images

\begin{abstract}
Through NSCT transformation, an image will be decomposed into a low-pass sub-band and K-direction sub-bands, but the sparsity is different for each direction. Therefore, this paper proposes a new way for fusion of infrared and visible light images, which is based on improved compressive sensing. First, strengthen the infrared image. Then, making a NSCT decomposition to the enhanced infrared image and visible light image, next doing compression to high frequency subbands by improved compressive sensing, and after that to fuse them. For the low-pass sub-band, it uses block DCT of high frequency energy rule to fuse them. Finally, it gets fusion image by reconstruction of compressive sensing and inverse NSCT transform for data which has been fused. When compared with the traditional compressive Sensing method, the simulation shows that it not only improves parameters, such as entropy, and standard deviation, average gradient, but reduces the amount of data and running time effectively.
\end{abstract}

\section{Introduction}

Image fusion is using some algorithm of multiple sensors on the same target information of multiple images to integrate and extract, so as to synthesize more accurate, comprehensive and detailed image than single sensor image. With the development of science and technology, image fusion is widely used in the field of target recognition, medical image procession, remote sensing image procession, computer vision and so on.

Compressive Sensing theory (CS theory)[1] was proposed by Candès Tao and Donoho in 2006. The theory suggests to use the way much lower than the Nyquist sampling theorem to collect data, when the signal is compressible or sparse. Thus we can reconstruct the original signal accurately by using appropriate optimization algorithm. It breaks through the bottleneck of the Nyquist sampling theorem and makes the high resolution signal acquisition possible.

This paper puts forward the existed image fusion algorithm based on compressive sensing. Since the amount of computation of compressive sensing algorithm will rise exponentially with the increase of image scale, we use the features of the images to make a different compression and fuse them on the compressed domain. The simulation results show that we can achieve more effective image compression and better fusion effect by using a small amount of compression sampling data to fuse.

\section{NSCT transform method}

NSCT using nonsubsampled pyramid (NSP) structure to achieve multi-resolution decomposition by nonsubsampled directional filter bank (NSDFB) multi-directional decomposition. NSP will make up-sampling for all filters in this layer as the next filter group after the decomposition of each layer, and each layer meets the perfect reconstruction condition. The high frequency part is decomposed into direction subbands whose number is order 2 to any times. Every subband has the same size with source images, so NSCT transform has the translational invariance. 


\section{Compressive Sensing theory}

The CS theory proposed by Donoho and Candes is based on the condition that signal is sparse or compressive. It is a novel signal sampling, coding and decoding theory. For sparse signal $x \in R^{N}$ and a certain orthogonal basis $\Psi \in R^{N \times N}$, as every signa $x$ in space $R^{N}$ can be expressed as $x=\sum_{i=1}^{N} \theta_{i} \psi_{i}$ or $X=\Psi \theta$, where $\theta$ is a sparse matrix with $\mathrm{K}$ non-zero components $(\mathrm{K}<<\mathrm{N})$. If $x$ is sparse, we can get a measurement vector $y$ (with less dimension than $x)$ by designing a sensing matrix $\Phi(M \times N)$ for sampling. that is:

$$
y=\Phi x
$$

then we can recover the sparse signal $x$ by a recovery algorithm. For sensing matrix $\Phi$, it should satisfy restricted isometry properties (RIP)[2]. And Guassian matrix, Bernoulli matrix and random selected Fourier matrix are typical examples.These matrixes are uncorrelated with most sparse orthogonal basis and satisfy RIP. The recovery of sparse signal x can be presented as

$$
\min \|\theta\|_{0} \text { s.t } y=\Phi x=\Phi \Psi \theta
$$

(2) is a combination optimization problem. It is hard to solve, for it is an underdetermined equation set. But Donoho indicates that if $\mathrm{x}$ is sparse, under a certain condition, the problem can be converted to a convex-optimization problem.[3] That is to say, under that certain condition, L-1-regularized are equivalent to L-0-regularized, so (2) can be converted to

$$
\min \|\theta\|_{1} \text { s.t } y=\Phi x=\Phi \Psi \theta
$$

Using recovery algorithm to solve (3), we can recover signal $x$ by inverse transform. There are two sorts of recovery algorithms. First, it converts combination optimization problem based on $L-0$ regularized to $L$-1-regularized, such as BP algorithm proposed by Donoho. Second, it converts combination optimization problem based on $L$-0-regularized to $L$-2-regularized, such as OMP,SP and CoSaMP developed by Wei Dai.

\section{The improved CS_NSCT image fusion method}

In the fusion process, due to there are too many subband coefficients involve in bandpass, the traditional band-pass sub-band fusion algorithm has many problems. Such as too large amount of calculation, the slow speed of operation and poor effect of fusion.Therefore, according to the characteristics of NSCT decomposition coefficient, this paper puts forward an improved fusion image method named CS_NSCT. We calculate the sparse degree K of high frequency coefficient in each part according to the NSCT decomposition , setting different values to change the sampling rate. Then we calculate every part of the measurement for $\mathrm{M}$, constructing the $M \times N$ measurement matrix $\Phi$ for each layer and each direction of high frequency coefficient, and $\mathrm{N}$ is the number of high frequency coefficient for a certain direction. The last we use linear measurements of the highfrequency coefficients to obtain the measurements value $Y_{K}$.

The improved image fusion algorithm based on CS_NSCT transform steps are as follows:

Step 1: Strict registration of infrared image and visible light image, and contrast enhancement of infrared image.The original infrared image and enhanced infrared image are Fig.1 (a), (b). 


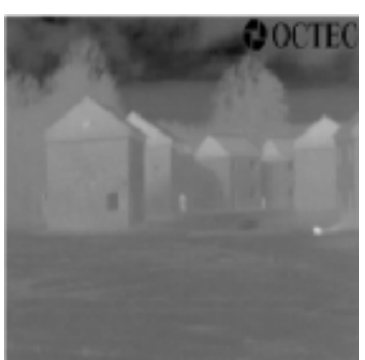

(a) The original infrared image

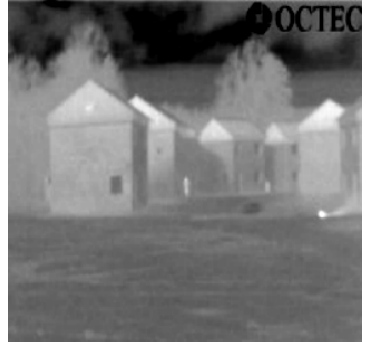

(b) The enhancement Infrared image

Fig. 1: Infrared image after registration

Step2:Make three layers of NSCT decomposition to the enhancement infrared image and visible light image, geting the high frequency subband and the low frequency subband respectively.

Step3: Fusing the NSCT low frequency. Due to the hot targets have higher frequency in infrared image while it is backgrounds in visible image.Therefore, when fusion we use infrared image coefficients in hot targets while visible image coefficients in backgrounds Considering this requirement, this paper uses the block DCT high frequency energy rules[4] .The criterion based on the DCT transform coefficient matrix, in which the low frequency coefficients are concentrated in the upper left corner, and high frequency coefficients to the lower right corner. It chooses the fusion coefficients according to the contrast of high frequency energy. If calculate the energy of the DCT block directly in which the high frequency energy will easily be masked by low frequency energy, but coefficients on the right side of deputy diagonal in block can basically reflect the high frequency information in source image. So we define the high frequency energy in DCT transformation matrix as follows.

$$
E=\sum_{u=0}^{N-1} \sum_{N-u-1}^{N-1} D(u, v)^{2}
$$

Where $\mathrm{N}$ is the image block size, $D(u, v)$ is coefficients of DCT coefficient matrix. In the fusion process, there is part of gray uniform areas in source image. Meanwhile, if just by high frequency energy of DCT coefficients for fusion, it will make miscalculation, and the region usually is background. Thus, if the difference value of DCT high frequency energy for two source images is less than the threshold $\mathrm{T}$ when fusion, coefficients of visible image has a high weight in fusion, as follows:

$$
X_{F}(i, j)=0.9 \times X_{A}(i, j)+0.1 \times X_{B}(i, j) \quad(0 \leq i, j \leq N-1)
$$

Where $X_{F}(i, j)$ is the coefficient after fusion, $X_{A}(i, j)$ and $X_{B}(i, j)$ are pixels for two source image. In the case of the difference value is greater than $T$, we will choose the visible image coefficients if visible image energy is bigger, otherwise, the infrared image coefficients are chosen. As following formula shows:

$$
X_{F}(i, j) \begin{cases}a \times X_{A}(i, j)+(1-a) \times X_{B}(i, j) & E_{A}-E_{B}>T \\ b \times X_{A}(i, j)+(1-b) \times X_{B}(i, j) & E_{A}-E_{B}<-T\end{cases}
$$

Where $\mathrm{a}, \mathrm{b}$ is weights under two different cases, in this paper $\mathrm{a}=0.9, \mathrm{~b}=0.3 . E_{A}, E_{B}$ represent the DCT block energy coefficient matrix of visible image and infrared image respectively.

Step4: The high frequency component will select the bigger sparse degree $\mathrm{K}$, through the calculation of different sparsity $\mathrm{K}$ at each layer for every directional sub-band. It will determine the measured value $M \geq c K \log (N / K)$ [5] ,where can change the sampling rate with the setting for constant $\mathrm{c}$ (c is a very small constant[6]) and get the corresponding value of $\mathrm{M}$. Then it will obtain the measurement matrix with size $M \times N$. Finally, it makes fusion with absolute value criteria in the compressed domain.

Where $Y_{K}(i, j)$ is the measurement value of two source image , $Y_{F}(i, j)$ is the measurement value of fused image.Then the fusion of high frequency coefficients measured value is:

$$
Y_{F}(i, j)=Y_{M}(i . j) \text { with } \arg \max _{K=A, B}\left(\left|Y_{K}(i, j)\right|\right)
$$


Step5: Using the Orthogonal matching pursuit algorithm to reconstruct the high frequency image after compression and fusion.

Step6: Do the inverse NSCT transformation for the reconstructed high frequency fused image and low frequency fused image.Finally we will obtain the fused image .

\section{The experimental results and analysis}

This paper makes a comparison with other algorithms in the view of objective and subjective evaluation, which aims to verify the validity of algorithm put in this paper.

\subsection{The objective evaluation parameters}

From the perspective of objective evaluation, it uses the information entropy, standard deviation, and average gradient evaluation to compare different algorithms. Following is many evaluation standards which are used commonly:

i) Information Entropy (E): Image information entropy is one important indicator to measure image information richness. Entropy reflects the average amount of information in the image. The greater the value of entropy, the more the information contained, and the fusion quality is better.The information entropy of image is defined as follow:

$$
E=-\sum_{i=1}^{n} P_{i} \log _{2} P_{i}
$$

Where $P_{i}$ is the probability distribution of image gray level of $\mathrm{i}, \mathrm{n}$ is the total number of gray level.

ii) Standard Deviation (SD): It instructions the discrete situation of image relative to the average grey value. The bigger the standard deviation, the more dispersed of the image grayscale distribution, and the fusion result is better. The image standard deviation is defined as:

$$
S T D=\sqrt{\frac{\sum_{i=0}^{M-1} \sum_{j=0}^{N-1}(F(i, j)-\bar{F})^{2}}{M \times N}}
$$

Where $F(i, j)$ represents the gray value of point $(i, j)$ in fusion image, and $\bar{F}$ is the average grey value of whole fusion image.

iii) Average Gradient (Ag): The average gradient can reflect the clarity of fusion image, it gives the tiny details of contrast and texture characteristics in images.The average gradientis of image is defined as:

$$
A G=\frac{1}{(M-1)(N-1)} \times \sum_{i=1}^{M-1} \sum_{j=1}^{N-1} \sqrt{\frac{(f(i+1, j))-F(i, j)^{2}+(F(i, j+1)-F(i, j))^{2}}{2}}
$$

iv) Time (t): It uses the functions in Maltab, such as tic and toc to compute the time.

\subsection{The simulation results}

Based on Matlab7.1 platform, it selects the visible light and infrared image to make experiments and analysis, where every picture is $256 \times 256$ grayscale and has been registered. In addition, the fusion experiments were conducted by NSCT method, the traditional CS_NSCT method, and the method put forward in this paper. It assesses the fusion effects from the aspects of subjective and objective.

As shown in Fig. 2, it's the simulation effect of fusion. We will make a three layers of NSCT decomposition for the visible light image and enhanced infrared image respectively, there Fig. 2(c) is the fusion effect who only do the NSCT decomposition, and figure (d) is the result of using traditional compression perception, but figure (e) is the effect of method in this paper. From subjective perspective, three ways all can extract the heat source information from infrared image, and show features in fusion image belong to visible light image or infrared image. 
The objective parameters for three algorithms are shown in table 1. As it says, firstly, the algorithm in this paper plays a good role in E, Ag, SD from the fusion effects. Secondly, the proposed way is obviously better than the NSCT way, and only takes one over ten times as long as NSCT. Finally, in the view of data amount, with the smaller number of sampling points (M), the measurement matrix will smaller, and the data after compressed will less. While traditional CS_NSCT uses the same compression for sub-bands, this paper makes compression based on sparsity, where the number of sampling points is between 150 and 180. Setting the sampling point to 180 in traditional CS_NSCT algorithm, the compressed data will be 562K after high frequency sub-bands compression by the way in this paper, and the data in traditional CS_NSCT is 645K, which means the data amount reduced by about $13 \%$ after compression. This way not only cut down the data amount needed in high frequency fusion, but the number of data in fusion and reconstruction is less. This advantage has a clear display as the image size and the number of decomposition layers increase. In conclusion, this way can effectively save the cost for transport, storage and reconsitution under the same quality of fused image.



(a)

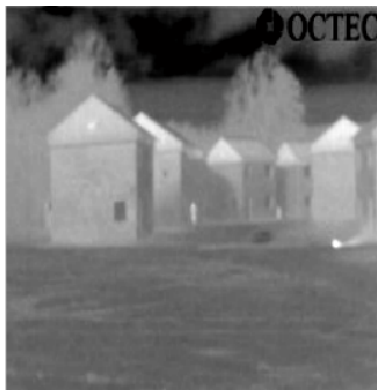

(b)

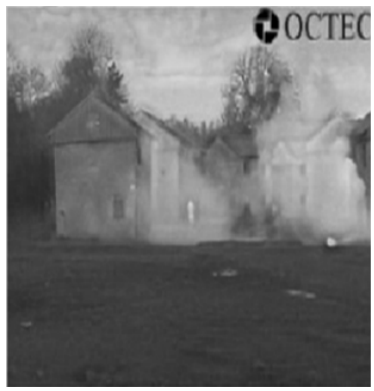

(c)

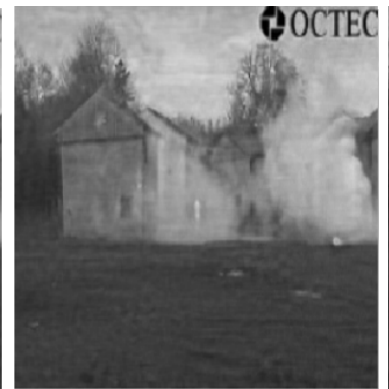

(d)



(e)

(a) the visible light image ,(b) the enhanced infrared image ,(c) the fusion image by NSCT ,(d) the fusion image by traditional CS_NSCT,(e) the fusion image of algorithm in this paper

Fig.2: the simulation results of image fusion

Table 1:The objective parameters of comparison of three algorithms for image Fusion

\begin{tabular}{|c|c|c|c|c|c|}
\hline Method & Time/s & Data size/K & E & Ag & SD \\
\hline $\begin{array}{c}\text { NSCT fusion } \\
\text { algorithm }\end{array}$ & 236.4 & 917 & 6.9950 & 4.2436 & 49.0433 \\
\hline $\begin{array}{c}\text { The traditional } \\
\text { CS_NSCT fusion } \\
\text { algorithm }\end{array}$ & 26.7 & 645 & 6.9218 & 3.9600 & 47.0350 \\
\hline $\begin{array}{c}\text { Algorithm in this } \\
\text { paper }\end{array}$ & 20.6 & 562 & 6.9783 & 4.1299 & 47.0395 \\
\hline
\end{tabular}




\section{Conclusions}

According to the characteristics of compressed sensing, this paper puts it into application in the field of image fusion effectively. It makes preprocessing to the infrared image, next does NSCT decomposition for infrared image, which has been processed, and visible light image. Then it uses the improved compression method for compressible high-frequency images to replace the traditional compression perception method. The simulation results show that this algorithm can significantly reduce the amount of calculating data, shorten the processing time, and got a better fusion effect.

\section{Acknowledgements}

The work is supported by the National Natural Science Foundation of China (Grant No. 61302133),Scientific and Technological Research Project of Science and Technology Department of Shaanxi Province (Grant Nos. 2011K09-36 and 2012K06-16) and Special project of Education Department of Shaanxi Province (Grant No. 12JK0528).

\section{References}

[1] Donoho D.L. Compressed sensing[J]. IEEE Transactions on Information Theory, 52(4),pp.1289-1306,2006

[2] De Castro L N,Von Zuben F. The clonal selection algorithm with engineering applications[C].Proc of Genetic and Evolutionary Computation Conference.USA:Morgan kaufman Publishers,pp,36_37,2000:

[3] Needell D,Vershynin R .Uniform uncertaintyprinciple and signal recovery cia regularized orthogonal matching pursuit [J].Foundations of Computational Mathematics,9(3),pp.317334,2009

[4] Cai xi. Multiscale image fusion. Beijing, Publishing House of Electronics Industry,1-159.2014

[5] Riehard Baraniuk, Mark Davenport, Ronald DeVore, Michael Wakin, A simple proof of the restricted Isometry Property for random matrices[J]. Constructive Approximation, Dee.28(3),pp.253-263,2008

[6] Candès E J , Tao T. Decoding by linear programming[J]. IEEE Transactions on Information Theory,51(12),pp.4203-4215, 2005 